\title{
A scheme for estimating the location and tangential plane of a reflector
}

\author{
Hiroshi Takenaka \\ Department of Earth and Planetary Sciences, Kyushu University, Hakozaki 6-10-1, Fukuoka 812-8581, Japan
}

(Received August 23, 1999; Revised February 20, 2000; Accepted March 10, 2000)

\begin{abstract}
Magmatic reflectors have been detected beneath some volcanic regions by analysing some reflection phases of seismograms. In this letter we consider a problem to determine the location and tangential plane of such a reflector from the ray parameter, back-azimuth and travel time of a reflection phase, and present a scheme to solve it. It is then shown that the problem is essentially a one-dimensional root-finding problem whose independent variable is only the depth of the reflection point, and thus it can be solved by a line search method such as the one-dimensional grid search. While homogeneous velocity structure models have been used in the conventional analyses, the present scheme employs a vertically inhomogeneous one and can also easily treat converted phases at the reflector.
\end{abstract}

\section{Introduction}

Last two decades magmatic reflectors have been detected beneath some volcanic regions by analysing reflection phases of seismograms (e.g., Mizoue, 1980; Mizoue and Ishiketa, 1988; Horiuchi et al., 1988; Hori and Hasegawa, 1991; Matsumoto and Hasegawa, 1996). Approaches that have been employed for the detection of these reflectors in the previous studies may be grouped into the following:

(1) Reflector is assumed to be a single horizontal plane, and its depth is estimated from travel time data of a reflection phase observed at all stations for all events (e.g., Sanford et al., 1973; Mizoue, 1980; Iwase et al., 1989; Hartse et al., 1992; Balch et al., 1997).

(2) Reflector's tangential plane at each reflection point is approximated by a horizontal plane to determine the location of a reflection point for the reflection phase for each event-station pair, and the entire geometry of the reflector, whose shape is usually assumed to be a single inclined plane, is estimated from the spatial distribution of the reflection points (e.g., Sanford et al., 1973; Mizoue et al., 1982; Mizoue and Ishiketa, 1988; Inamori et al., 1992).

(3) Reflector is assumed to be a single inclined flat plane, and its location, dip direction and dip angle are estimated by use of all available travel time data of reflection phases in combination with hypocenter coordinates (Sanford et al., 1973; Horiuchi et al., 1988; Hori and Hasegawa, 1991, 1999).

(4) Curved reflector surface is approximated by ensemble of many small inclined planes (like patchwork) which are determined from travel time data of reflection phases for different combinations of station and hypocenters (Matsumoto and Hasegawa, 1996, 1997).

Copy right (c) The Society of Geomagnetism and Earth, Planetary and Space Sciences (SGEPSS); The Seismological Society of Japan; The Volcanological Society of Japan; The Geodetic Society of Japan; The Japanese Society for Planetary Sciences.
In the above four approaches, the main attribute of observed data used for analysis is the travel time of reflection phases. In most studies employing the approaches, velocity structure was assumed to be homogeneous above the reflector in the calculation of ray paths because of simplicity, although it is possible to relax this assumption (e.g., Hartse et al., 1992; Balch et al., 1997).

In this short note we consider a different approach from the previous studies mentioned above. We consider a problem (situation) to determine the location of a reflection point and the tangential plane touching the reflector surface at the reflection point from the ray parameter (inverse of apparent velocity) and back-azimuth of a reflection phase as well as the travel time, and show a simple algorithm to solve it. The ray parameter and back-azimuth can in practice be evaluated, for example, by an array analysis such as the semblance analysis (e.g., Nikolaev and Troitskiy, 1987; Korn, 1988; Kuwahara et al., 1990). In the present algorithm, vertically inhomogeneous (layered) velocity models are employed, and reflected converted phases can be also easily treated. For convenience, we hereafter call the tangential plane of the reflector at the reflection point the "reflector facet".

\section{Problem Setting}

Throughout this note we employ a right handed Cartesian coordinate system $(x, y, z)$, and take coordinate directions $[x, y, z]$ as [North, East, Down]. Further we set the origin (O) of the coordinate system to be at the observation point on the ground where the ray parameter, back-azimuth and travel time of a reflection phase have been measured. In case of array observation, the origin of array can be chosen as the observation point.

Figure 1 shows the geometry of the problem to be solved. Points $\mathrm{S}$ and $\mathrm{E}$ denote the hypocenter and epicenter of a source, respectively, and $\mathrm{R}$ and $\mathrm{P}$ are the reflection point and its projection onto the plane of $z=0$ (i.e., ground surface). Vector $\boldsymbol{n}$ is the upward unit normal to the reflector facet. Vector $\boldsymbol{p}$ is the horizontal slowness vector of the reflected 


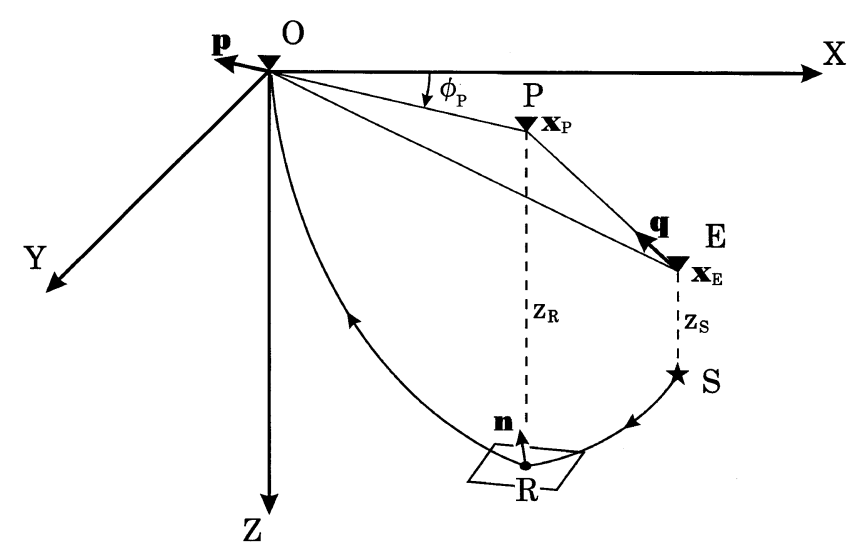

Fig. 1. Configuration of the problem to be solved. Points $\mathrm{S}$ and $\mathrm{E}$ are the source and its epicenter. $\mathrm{R}$ and $\mathrm{P}$ are the reflection point and its projection onto the plane of $z=0$ (ground surface). $\boldsymbol{n}$ is the upward normal vector of the reflector facet. $\boldsymbol{p}$ and $\boldsymbol{q}$ are the horizontal slowness vector at the observation point and at the source, respectively. $\phi_{\mathrm{P}}$ is the back-azimuth of the reflection phase observed at $\mathrm{O}$ (i.e., the back-azimuth of $\boldsymbol{p}$ ).

ray which is defined by the measured ray parameter $(p)$ and back-azimuth $\left(\phi_{\mathrm{P}}\right)$ of the reflection phase observed at the observation point $\mathrm{O}$ as follows:

$$
\boldsymbol{p}=\left(-p \cos \phi_{\mathrm{P}},-p \sin \phi_{\mathrm{P}}, 0\right)^{\mathrm{T}},
$$

where $\mathrm{T}$ denotes the transpose of vector.

In the problem we will find the coordinates of the reflection point $\mathrm{R}$ (or the position $\left(\boldsymbol{x}_{\mathrm{P}}\right)$ of point $\mathrm{P}$ and the depth $\left(z_{\mathrm{R}}\right)$ of the reflector point) and the normal vector of the reflector facet $\boldsymbol{n}$ when we know the position $\left(\boldsymbol{x}_{\mathrm{E}}\right)$ of the epicenter $\mathrm{E}$ and depth $\left(z_{\mathrm{S}}\right)$ of a source, travel time $\left(T_{\text {data }}\right)$ and horizontal slowness vector $\boldsymbol{p}$ of the reflection phase observed at $\mathrm{O}$ as well as the subsurface velocity structure. For convenience, we separate the subsurface velocity structure into the following two: one is the velocity profile $V_{(\mathrm{S})}=V_{(\mathrm{S})}(z)$ of a seismic phase ( $P$ - or $S$-wave velocity) along the ray from the source $\mathrm{S}$ to the reflection point $\mathrm{R}$, and the other is the velocity profile $V_{(\mathrm{O})}=V_{(\mathrm{O})}(z)$ along the ray from $\mathrm{R}$ to the observation point $\mathrm{O}$. When we consider converted phases at the reflector, $V_{(\mathrm{S})}$ and $V_{(\mathrm{O})}$ are different. In case of $S P$ reflection ( $S \times P$ phase), for instance, $V_{(\mathrm{S})}$ is a $S$-wave velocity profile, while $V_{(\mathrm{O})}$ is a $P$-wave one. Even in case of unconverted reflection $(P \times P$ or $S \mathrm{x} S$ phase), we can employ different velocity profiles of $V_{(\mathrm{S})}$ and $V_{(\mathrm{O})}$ for shallow parts above R, so that we can take account of different site conditions between around the source and the receiver.

\section{Algorithm for Solution}

In this section we show that the problem set up in the previous section can be solved by a line search for $z_{\mathrm{R}}$ to describe a solution algorithm. There are several methods for a line search, for example, the one-dimensional grid search, the bisection method and the secant method (e.g., Press et al., 1992). In this section we assume the use of the simplest line search method, the one-dimensional grid search, to make the structure of the solution algorithm show up although any more sophisticated methods for line search are also applicable to the solution algorithm.
Setting a trial value to $z_{\mathrm{R}}$, which must satisfy

$$
1 / V_{(\mathrm{O})}(z)>p \quad \text { for } \quad z \leq z_{\mathrm{R}} \text {, }
$$

the distance between the observation point $\mathrm{O}$ and point $\mathrm{P}$, $r_{\mathrm{P}} \equiv \overline{\mathrm{OP}}$, associated with the trial value of $z_{\mathrm{R}}$ is calculated by

$$
r_{\mathrm{P}}=X\left(p, 0, z_{\mathrm{R}}\right)
$$

where

$$
\begin{aligned}
& X\left(p, z, z^{\prime}\right)= \frac{p V^{(n)}\left(z_{n}-z\right)}{\sqrt{1-\left(p V^{(n)}\right)^{2}}}+\sum_{i=n+1}^{n^{\prime}-1} \frac{p V^{(i)} h^{(i)}}{\sqrt{1-\left(p V^{(i)}\right)^{2}}} \\
&+\frac{p V^{\left(n^{\prime}\right)}\left(z^{\prime}-z_{n^{\prime}-1}\right)}{\sqrt{1-\left(p V^{\left(n^{\prime}\right)}\right)^{2}}}, \\
& z_{n-1}<z<z_{n}, \quad z_{n^{\prime}-1}<z^{\prime}<z_{n^{\prime}} .
\end{aligned}
$$

$X\left(p, z, z^{\prime}\right)$ is the horizontal distance over which a seismic phase travels along the ray with ray parameter $p$ from a depth level $z$ to a deeper level $z^{\prime}$ (or from $z^{\prime}$ to $z$ ), where the levels $z$ and $z^{\prime}$ are in the $(n)$-th and the $\left(n^{\prime}\right)$-th layer, respectively (Fig. 2). $V^{(i)}$ is the velocity of the considered phase in the (i)-th layer whose thickness is $h^{(i)}$. Equation (3) uses those of the velocity profile $V_{(\mathrm{O})}$ along the reflected (or reflected converted) ray from the reflection point $\mathrm{R}$ to the observation point $\mathrm{O}$ for $V^{(i)}$ and $h^{(i)}$. Since point $\mathrm{P}$ must fall on the line which is through $\mathrm{O}$ and parallel to $\boldsymbol{p}$, the position $\boldsymbol{x}_{\mathrm{P}}$ of point $\mathrm{P}$ (i.e., the horizontal coordinates of the reflection point $\mathrm{R}$ ) is derived as

$$
\boldsymbol{x}_{\mathrm{P}}=\left(r_{\mathrm{P}} \cos \phi_{\mathrm{P}}, r_{\mathrm{P}} \sin \phi_{\mathrm{P}}, 0\right)^{\mathrm{T}} .
$$

Note that this derived horizontal position $\boldsymbol{x}_{\mathrm{P}}$ of the reflection point is corresponding to the selected trial value of $z_{\mathrm{R}}$. The condition that the chosen value of $z_{\mathrm{R}}$ and the derived position $\boldsymbol{x}_{\mathrm{P}}$ is the solution of the problem set up in the previous section, is that the corresponding two-way travel time of the reflection phase calculated assuming them is equal to the measured

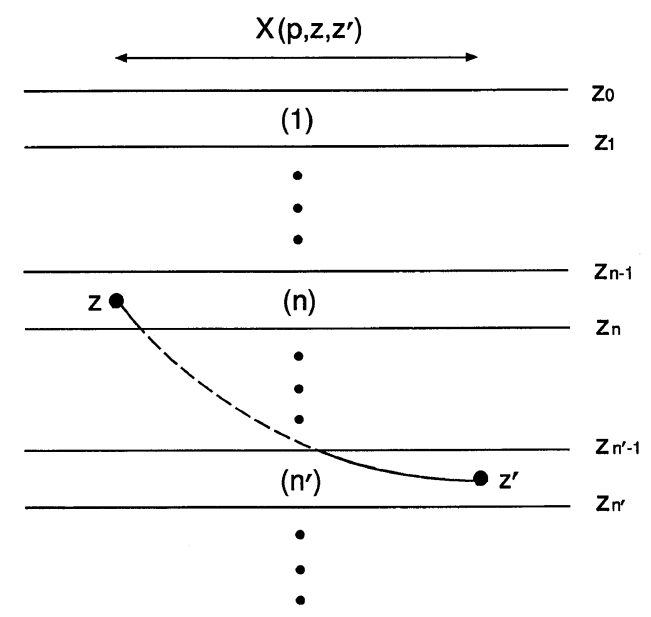

Fig. 2. Configuration of velocity model for Eqs. (4) and (7). Point at a depth level $z$ and point at another level $z^{\prime}\left(z<z^{\prime}\right)$, between which horizontal distance is $X\left(p, z, z^{\prime}\right)$, are connected by a ray with ray parameter $p$. One-way travel time along this ray from level $z$ to level $z^{\prime}$ (or from level $z^{\prime}$ to level $\left.z\right)$ is $T\left(p, z, z^{\prime}\right)$. 
travel time $T_{\text {data }}$. Next we describe a scheme to calculate the two-way travel time of the reflection phase.

The two-way travel time is the sum of the travel time $\left(T_{\mathrm{SR}}\right)$ from the source $\mathrm{S}$ to the reflection point $\mathrm{R}$ and that $\left(T_{\mathrm{RO}}\right)$ from $\mathrm{R}$ to the observation point $\mathrm{O}$. The latter is obtained as

$$
T_{\mathrm{RO}}=T\left(p, z_{\mathrm{O}}, z_{\mathrm{R}}\right)
$$

where

$$
\begin{aligned}
& T\left(p, z, z^{\prime}\right)= \frac{z_{n}-z}{V^{(n)} \sqrt{1-\left(p V^{(n)}\right)^{2}}} \\
&+\sum_{i=n+1}^{n^{\prime}-1} \frac{h^{(i)}}{V^{(i)} \sqrt{1-\left(p V^{(i)}\right)^{2}}} \\
&+\frac{z^{\prime}-z_{n^{\prime}-1}}{V^{\left(n^{\prime}\right)} \sqrt{1-\left(p V^{\left(n^{\prime}\right)}\right)^{2}}}, \\
& z_{n-1}<z<z_{n}, \quad z_{n^{\prime}-1}<z^{\prime}<z_{n^{\prime}} .
\end{aligned}
$$

Equation (6) employs the velocity profile $V_{(\mathrm{O})}$ along the reflected (or reflected converted) ray from $\mathrm{R}$ to $\mathrm{O}$ for $V^{(i)}$ and $h^{(i)}$ in Eq. (7).

To compute $T_{\mathrm{SR}}$, it is necessary to find the ray parameter (q) of the ray at source which is invariant along the path from the source $S$ to the reflection point $R$. This can be done by solving the following equation for $q$ with the same formulation as Eq. (4):

$$
X\left(q, z_{\mathrm{S}}, z_{\mathrm{R}}\right)=\left|\boldsymbol{x}_{\mathrm{P}}-\boldsymbol{x}_{\mathrm{E}}\right|,
$$

where $V^{(i)}$ and $h^{(i)}$ in Eq. (4) for the left hand side of this equation are those of the velocity profile $V_{(\mathrm{S})}$ along the ray path from $\mathrm{S}$ to R. Equation (8) can be easily solved numerically by a line search scheme such as the one-dimensional grid search. If Eq. (8) does not have any real root, we select a new trial value of $z_{\mathrm{R}}$ which is less than the original one, and restart from Eq. (3).

Travel time $T_{\mathrm{SR}}$ from the source $\mathrm{S}$ to the reflection point $\mathrm{R}$ is calculated with Eq. (7) by

$$
T_{\mathrm{SR}}=T\left(q, z_{\mathrm{S}}, z_{\mathrm{R}}\right),
$$

where the velocity profile $V_{(\mathrm{S})}$ is employed. The two-way travel time $\left(T_{\mathrm{SRO}}\left(z_{\mathrm{R}}\right)\right)$ of the reflection phase associated with the selected trial value of $z_{\mathrm{R}}$ is then

$$
T_{\mathrm{SRO}}\left(z_{\mathrm{R}}\right)=T_{\mathrm{SR}}\left(z_{\mathrm{R}}\right)+T_{\mathrm{RO}}\left(z_{\mathrm{R}}\right) .
$$

If this calculated value of $T_{\mathrm{SRO}}$ is equal to the measured travel time $T_{\text {data }}$ (in practice the difference between them is less than a pre-specified tolerance), we regard the chosen trial value of $z_{\mathrm{R}}$ and the calculated position $\boldsymbol{x}_{\mathrm{P}}$ as the solution, and use them to derive the last unknown quantity of the problem, the normal vector of the reflector facet $\boldsymbol{n}$. Otherwise, we select a next trial value of $z_{\mathrm{R}}$ along a rule of the grid search to restart from Eq. (3), and iterate the process described above until we find a value of $z_{\mathrm{R}}$ which we can regard as the solution.

Upward normal vector $\boldsymbol{n}$ of the reflector facet is obtained from Snell's law for reflection, that is, the tangential component of the slowness vector of the reflected (or reflected converted) ray at the reflection point is equal to that of the incident ray on the reflector facet just before the reflection:

$$
\boldsymbol{n}=\frac{\boldsymbol{P}-\boldsymbol{Q}}{|\boldsymbol{P}-\boldsymbol{Q}|}
$$

where $\boldsymbol{P}$ and $\boldsymbol{Q}$ are the slowness vector of the reflected (or reflected converted) ray (upgoing ray) at the reflection point and that of the incident ray (downgoing ray) on the reflector facet just before the reflection (reflection conversion), respectively (Fig. 3). $\boldsymbol{P}$ and $\boldsymbol{Q}$ can be derived from the quantities known so far by

$$
\boldsymbol{P}=\left(p_{x}, p_{y},-v\right)^{\mathrm{T}}, \quad \boldsymbol{Q}=\left(q_{x}, q_{y}, \xi\right)^{\mathrm{T}},
$$

where $p_{x, y}$ and $q_{x, y}$ are $x$ - and $y$-component of $\boldsymbol{p}$ and the following vector $\boldsymbol{q}$ :

$$
\boldsymbol{q}=q\left(\boldsymbol{x}_{\mathrm{P}}-\boldsymbol{x}_{\mathrm{E}}\right) /\left|\boldsymbol{x}_{\mathrm{P}}-\boldsymbol{x}_{\mathrm{E}}\right|
$$

This is the horizontal slowness vector of the ray at source (see Fig. 1). The vertical component of $\boldsymbol{P}$ and $\boldsymbol{Q},-v$ and $\xi$ are calculated as

$$
v=\sqrt{\left[1 / V_{(\mathrm{O})}\left(z_{\mathrm{R}}\right)\right]^{2}-p^{2}}, \quad \xi=\sqrt{\left[1 / V_{(\mathrm{S})}\left(z_{\mathrm{R}}\right)\right]^{2}-q^{2}} .
$$

\section{Discussion and Summary}

In the previous sections we have set up a problem to determine the position of the reflection point and the normal vector of the reflection facet of a reflected (or reflected converted) phase from the travel time, ray parameter and back-azimuth of the reflection phase, and have shown an algorithm to find the solution. The algorithm is summarised as follows:

[1] Select a trial value of $z_{\mathrm{R}}$.

[2] Calculate the horizontal position $\boldsymbol{x}_{\mathrm{P}}$ by Eqs. (3) to (5).

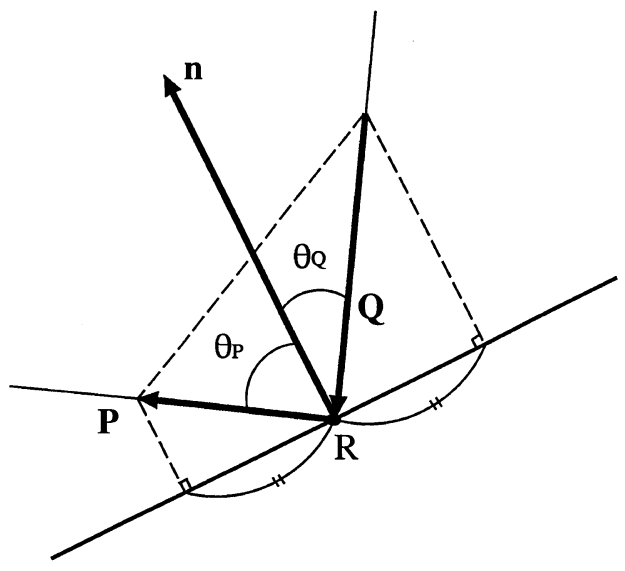

Fig. 3. Reflection (reflection conversion) at a reflector facet. $\boldsymbol{Q}$ is the slow ness vector of the incident ray at the reflection point $\mathrm{R}\left(|\boldsymbol{Q}|=1 / V_{(\mathrm{S})}\left(z_{\mathrm{R}}\right)\right)$, while $\boldsymbol{P}$ is that of the reflected (or reflected converted) ray at $\mathrm{R}$ $\left(|\boldsymbol{P}|=1 / V_{(\mathrm{O})}\left(z_{\mathrm{R}}\right)\right)$. Angles $\theta_{Q}$ and $\theta_{P}$ that the incident and the reflected rays make with the upward normal vector $(\boldsymbol{n})$ of the reflection facet are governed by Snell's law: $|\boldsymbol{P}| \sin \theta_{P}=|\boldsymbol{Q}| \sin \theta_{Q}$. In case of the reflection without conversion, $\theta_{P}=\theta_{Q}$; while in case of the reflection with conversion, $\theta_{P} \neq \theta_{Q}$. Note that in both cases $\boldsymbol{n}$ is parallel to the composite vector $\boldsymbol{P}-\boldsymbol{Q}$. 
[3] Find the ray parameter $q$ of the ray at source by solving Eq. (8). If Eq. (8) does not have any real root, select a new trial value of $z_{\mathrm{R}}$ which is less than the original one, and return to step [2].

[4] Calculate the two-way travel time of the reflection phase $T_{\text {SRO }}$ using Eqs. (6), (7), (9) and (10).

[5] Compare the calculated travel time $T_{\mathrm{SRO}}$ with the measured one $T_{\text {data }}$ to determine whether the trial value of $z_{\mathrm{R}}$ and the $\boldsymbol{x}_{\mathrm{P}}$ calculated in step [2] are the desired solution or not. If they are regarded as the solution, proceed to the next step [6]. Otherwise, select a next trial value of $z_{\mathrm{R}}$ along the rule of a line search method, and return to step [2].

[6] Calculate the normal vector of the reflection facet $\boldsymbol{n}$ by Eqs. (11) to (14).

This algorithm clearly shows that the depth of the reflection point $z_{\mathrm{R}}$ is only an independent variable of the problem considered here, and therefore it is essentially a one-dimensional root finding problem (e.g., Press et al., 1992).

The derived position of the reflection point and normal vector of the reflector facet gives the equation of the reflector facet, which is represented as

$$
n_{x}\left(x-r_{\mathrm{P}} \cos \phi_{\mathrm{P}}\right)+n_{y}\left(y-r_{\mathrm{P}} \sin \phi_{\mathrm{P}}\right)+n_{z}\left(z-z_{\mathrm{R}}\right)=0,
$$

where $n_{x, y, z}$ are $x$-, $y$ - and $z$-component of the normal vector $\boldsymbol{n}$. The dip angle $\delta$ and dip direction $\varphi$ of the facet are also obtained as

$$
\delta=\cos ^{-1}\left(\left|n_{z}\right|\right),
$$

and

$$
\varphi=\operatorname{atan}\left(n_{y}, n_{x}\right),
$$

where the definition of the dip angle and dip direction of the reflector facet is the same as that for fault plane employed by Aki and Rechards (1980) (see figure 4.13 in Aki and Rechards (1980)). Further, the equation of intersection line of the facet and $x-z$ plane (North-South cross section) at the reflection point is expressed as

$$
\tan \delta \cos \varphi\left(x-r_{\mathrm{P}} \cos \phi_{\mathrm{P}}\right)-\left(z-z_{\mathrm{R}}\right)=0,
$$

and the equation of intersection line of the facet and $y-z$ plane (East-West cross section) at the reflection point is

$$
\tan \delta \sin \varphi\left(y-r_{\mathrm{P}} \sin \phi_{\mathrm{P}}\right)-\left(z-z_{\mathrm{R}}\right)=0 .
$$

The present new scheme for detecting reflector facets has been successfully applied to map a magmatic reflector beneath Unzen volcanic area, Shimabara Peninsula, Kyushu, Japan (Yamaguchi et al., 1999). Yamaguchi et al. (1999) analysed the $P \times P$ reflection phase appearing on the records of four small arrays for the explosion seismic experiment conducted at Unzen volcano in 1995 to investigate the structure of the volcano. They performed the semblance analysis for each combination of array and shot to estimate the ray parameter and back-azimuth of the reflection phase, and then applied the present scheme to the estimated values with the travel times. Using the same velocity model as employed for earthquake hypocenter determination routine around Unzen area, they found a reflector exists at a depth of about $15 \mathrm{~km}$ beneath the western shore of the Shimabara Peninsula and it has a slight dip down to the southwest, which is reasonable for the magma system model of Unzen volcano inferred by Umakoshi et al. (1994) from the seismicity. As this successful example shows, the present scheme is also available for seismic experiment data as well as natural earthquake data.

Acknowledgments. I gratefully acknowledge valuable discussions with Sosuke Yamaguchi and Prof. Sadaomi Suzuki. Atsushi Watanabe and Yanbin Wang kindly helped me make figures. I would like to thank Prof. Toshiro Tanimoto and two anonymous reviewers for critically reading the original manuscript and for providing helpful suggestions.

\section{References}

Aki, K. and P. G. Rechards, Quantitative Seismology: Theory and Methods, vol. 1, 557 pp., Freeman and Co., San Francisco, 1980.

Balch, R. S., H. E. Hartse, A. R. Sanford, and K. Lin, A new map of the geographic extent of the Socorro mid-crustal magma body, Bull. Seism. Soc. Am., 87, 174-182, 1997.

Hartse, H. E., A. R. Sanford, and J. S. Knapp, Incorporating Socorro magma body reflections into the earthquake location process, Bull. Seism. Soc. Am., 82, 2511-2532, 1992.

Hori, S. and A. Hasegawa, Location of a mid-crustal magma body beneath Mt. Moriyoshi, northern Akita Prefecture, as estimated from reflected SxS phases, Zisin (J. Seismol. Soc. Japan), Ser. II, 44, 39-48, 1991 (in Japanese with English abstract).

Hori, S. and A. Hasegawa, Distinct $S$-wave reflector detected in the uppermost mantle beneath Osoresan volcano, NE Japan, Bull. Volcanol. Soc. Japan, 44, 83-91, 1999 (in Japanese with English abstract).

Horiuchi, S., A. Hasegawa, A. Takagi, A. Ito, M. Suzuki, and H. Kameyama, Mapping of a melting zone near Mt. Nikko-Shirane in northern Kanto, Japan, as inferred from $S \mathrm{x} P$ and $S \mathrm{x} S$ reflections, Tohoku Geophys. J., 31, 43-55, 1988.

Inamori, T., S. Horiuchi, and A. Hasegawa, Location of mid-crustal reflectors by a reflection method using aftershock waveform data in the focal area of the 1984 Western Nagano Prefecture earthquake, J. Phys. Earth, 40, 379-393, 1992.

Iwase, R., S. Urabe, K. Katsumata, M. Moriya, I. Nakamura, and M. Mizoue, Mid-crustal magma body in southwestern Fukushima Prefecture detected by reflected waves from microearthquakes, Programme Abstr. Seismol. Soc. Japan., no. 1, 185, 1989 (in Japanese).

Korn, M., $P$-wave coda analysis of short-period array data and the scattering and absorptive properties of the lithosphere, Geophys. J., 93, 437-449, 1988.

Kuwahara, Y., H. Ito, M. Shinohara, and H. Kawakatsu, Small-array observation of seismic coda waves in Izu-Oshima-Analysis of coda waves from artificial explosions and from a natural intermediate-depth earthquake, Zisin (J. Seismol. Soc. Japan), Ser. II, 43, 359-371, 1990 (in Japanese with English abstract).

Matsumoto, S. and A. Hasegawa, Distinct $S$ wave reflector in the midcrust beneath Nikko-Shirane volcano in the northeastern Japan arc, J. Geophys. Res., 101, 3067-3083, 1996.

Matsumoto, S. and A. Hasegawa, Distribution of midcrustal $S$ wave reflectors beneath Nikko-Shirane volcano in the northeastern Japan arc, Bull. Volcanol. Soc. Japan, 42, 127-139, 1997 (in Japanese with English abstract).

Mizoue, M., Deep crustal discontinuity underlain by molten material as deduced from reflection phases on microearthquake seismograms, Bull. Earthq. Res. Inst., Univ. Tokyo, 55, 705-735, 1980.

Mizoue, M. and Y. Ishiketa, Detection of melting zone beneath fault area of the 1984 Western Nagano Prefecture earthquake and beneath the southeastern foot of Mt. Ontake, Gekkan Chikyu (Earth Monthly), 10, 700-705, 1988 (in Japanese).

Mizoue, M., I. Nakamura, and T. Yokota, Mapping of an unusual crustal discontinuity by microearthquake reflections in the earthquake swarm area near Ashio, northern part of Tochigi Prefecture, central Japan, Bull. Earthq. Res. Inst., Univ. Tokyo, 57, 653-686, 1982.

Nikolaev, A. V. and P. A. Troitskiy, Lithospheric studies based on array of $P$-coda and microseisms, Tectonophys., 140, 103-113, 1987.

Press, W. H., S. A. Teukolsky, W. T. Vetterling, and B. P. Flannery, Numerical Recipes in FORTRAN: The Art of Scientific Computing-2nd ed., 963 pp., 
Cambridge Univ. Press, New York, 1992.

Sanford, A. R., O. Alptekin, and T. R. Toppozada, Use of reflection phases on microearthquake seismograms to map an unusual discontinuity beneath the Rio Grande Rift, Bull. Seism. Soc. Am., 63, 2021-2034, 1973.

Umakoshi, K., H. Shimizu, and N. Matsuwo, Magma ascent path in the 1990-94 eruption of Fugendake, Unzen volcano, as inferred from precisely determined hypocentral distribution, Bull. Volcanol. Soc. Japan, 39, 223-235, 1994 (in Japanese with English abstract).
Yamaguchi, S., H. Takenaka, S. Suzuki, T. Murakoshi, and H. Shimizu, Mapping of a magmatic reflector beneath Unzen volcanic area as Inferred from $P \times P$ reflections in the 1995 explosion experiment, Abstr. 1999 Japan Earth Planet. Sci. Joint Meet., Vb-021, 1999 (in Japanese with English summary).

H. Takenaka (e-mail: takenaka@geo.kyushu-u.ac.jp) 